\title{
Comparative proteomics analysis reveals the difference during antler regeneration stage between red deer and sika deer
} \author{
Yang ${ }^{4}$, Daqing Zhao ${ }^{4}$, He Zhang ${ }^{\text {Corresp., } 3}$, Bin Qi ${ }^{\text {Corresp. } 2}$ \\ ${ }^{1}$ Practice Innovations Center, Changchun University of Chinese Medicine, Changchun, China \\ 2 College of Pharmacy, Changchun University of Chinese Medicine, Changchun, China \\ ${ }^{3}$ School of Clinical Medicine, Changchun University of Chinese Medicine, Changchun, China \\ 4 Jilin Ginseng Academy, Changchun University of Chinese Medicine, Changchun, China \\ Corresponding Authors: He Zhang, Bin Qi \\ Email address: 13843148162@163.com, qibin88@126.com
}

Hang Su ${ }^{1}$, Xiaolei Tang ${ }^{2}$, Xiaocui Zhang ${ }^{2}$, Li Liu $^{2}$, Li Jing ${ }^{1}$, Daian Pan ${ }^{3}$, Weijie Sun ${ }^{4}$, Huinan He ${ }^{4}$, Chonghui

Deer antler, as the only one mammalian regenerative appendage, provides an optimal model to study regenerative medicine. Antler harvested from red deer or sika deer was mainly study object to disclose the mechanism underlying antler regeneration mechanism over past decades. Previous study used proteomic technology to reveal the signaling pathways of antler stem cell derived from red deer. Moreover, transcriptome of antler tip from sika deer provide us the essential genes, which regulated antler development and regeneration. However, antler comparison between red deer and sika deer has not been well concerned. In current our study, proteomics was employed to analyze the biological difference of antler regeneration between sika deer and red deer. Proteomics profile was completed by searching UniProt database and differentially expressed proteins were identified by bioinformatic software. 36 proteins were highly expressed in red deer antler, while 144 proteins were abundant in sika deer. GO and KEGG analysis revealed that differentially expressed proteins participated in the regulation of several pathways including oxidative phosphorylation, ribosome, extracellular matrix interaction, and PI3KAkt pathway . 


\section{Comparative proteomics analysis reveals the difference during 2 antler regeneration stage between red deer and sika deer}

3 Hang Su ${ }^{1}$, Xiaolei Tang ${ }^{2}$, Xiaocui Zhang ${ }^{2}$, Li Liu ${ }^{2}$, Li Jing ${ }^{1}$, Daian Pan ${ }^{3}$, Weijie Sun ${ }^{4}$, Huinan He ${ }^{4}$,

4 Chonghui Yang ${ }^{4}$, Daqing Zhao ${ }^{4}$, He Zhang ${ }^{3, *}$ and Bin Qi ${ }^{2,{ }^{*}}$

$5{ }^{1}$ Practice Innovations Center, Changchun University of Chinese Medicine, Changchun 130117, China

$6{ }^{2}$ College of Pharmacy, Changchun University of Chinese Medicine, Changchun 130117, China

$7{ }^{3}$ School of Clinical Medicine, Changchun University of Chinese Medicine, Changchun 130117, China

84 Jilin Ginseng Academy, Changchun University of Chinese Medicine, Changchun 130117, China

9

10 Corresponding Author:

11 He Zhang and Bin Qi

12 Boshuo Rd. 1035, 130117, Changchun, Jilin, China

13 Email address: 13843148162@163.com (H.Z); qibin88@126.com (B.Q.)

14 
15

16

17

18

19

20

21

22

23

24

25

26

27

28

29

30

31

32

33

34

35

36

37

38

39

40

41

42

43

44

45

46

47

48

49

50

51

52

53

54

\section{Abstract}

Deer antler, as the only one mammalian regenerative appendage, provides an optimal model to study regenerative medicine. Antler harvested from red deer or sika deer was mainly study object to disclose the mechanism underlying antler regeneration mechanism over past decades. Previous study used proteomic technology to reveal the signaling pathways of antler stem cell derived from red deer. Moreover, transcriptome of antler tip from sika deer provide us the essential genes, which regulated antler development and regeneration. However, antler comparison between red deer and sika deer has not been well concerned. In current our study, proteomics was employed to analyze the biological difference of antler regeneration between sika deer and red deer. Proteome profile was completed by searching UniProt database and differentially expressed proteins were identified by bioinformatic software. 36 proteins were highly expressed in red deer antler, while 144 proteins were abundant in sika deer. GO and KEGG analysis revealed that differentially expressed proteins participated in the regulation of several pathways including oxidative phosphorylation, ribosome, extracellular matrix interaction, and PI3K-Akt pathway.

\section{Introduction}

Deer antlers are the mammalian regenerative organ, which is covered with abundantly vascular skin called velvet. The antler regeneration initiates in every spring (Fennessy 1984). At early regeneration stage, the main beam of antler grows dramatically on posterior growth center (Li et al. 2014). Subsequently, at fast regeneration stage, tines of antler will form on anterior growth center. During the antler regeneration stage, the growth rate of red antler will reach to $100 \mathrm{~g}$ per day within 100 to 120 days (Borsy et al. 2009). The weight of the full size of red deer antler generally reached to 7 to $9 \mathrm{~kg}$ (Borsy et al. 2009). However, the sika deer antler with 3 to $6 \mathrm{~kg}$ is less than red deer (Hu et al. 2019). Following into autumn, the antler gradually reaches the full size and then has calcification accompanied with the loss of velvet and vessels (Li et al. 2014). Over the past decades, this special feature attracted researchers to study on the development mechanism underlying antler regeneration. The antler generation through modified endochondral ossification was firstly reported by Banks and Newbrey (Banks \& Newbrey 1983). And antler histogenesis and tissue depletion study provided sufficient evidence that pedicle periosteum drive antler to regeneration ( $\mathrm{Li}$ et al. 2007; Li \& Suttie 1994; Li et al. 2004). Besides pedicle periosteum study, researches about the growing tip of antler with the fastest growth rate are also a hotspot (Deb-Choudhury et al. 2015; Gyurjan et al. 2007; Yao et al. 2012).

Molnár et al. identified two expression clusters for 36 genes that were mainly expressed in consecutive tissue zones of antler tip of red deer (Molnar et al. 2007). The first gene expression cluster was found to involve with ribosome pathway and the second cluster could be related with tumor biology. Furthermore, the activated runx 2 pathway up-regulated the expression of mineralization proteins in cartilaginous tissue of red deer antler (Steger et al. 2010). Stéger et al. demonstrated that antler regeneration model can be also used to study human osteoporosis (Steger et al. 2010). To further deeply study on deer antler, the systematic study of sika deer 
55

56

57

58

59

60

61

62

63

64

65

66

67

68

69

70

71

72

73

74

75

76

77

78

79

80

81

82

83

84

85

86

\section{Materials and Methods} antler growth.

\section{Tissue sampling}

antler tips based on transcriptome provided more information about the key regulated genes related to cartilage development (Yao et al. 2012). Li et al. firstly used proteomic technology to disclose the molecular mechanism of antler stem cells from red deer (Li et al. 2012a). This study identified the key markers of antler stem cells, which were transcription factors POU5F1, SOX2, NANOG, and MYC. Moreover, distinctive pathways involved in sika deer antler development were found through the comparative proteomics between potentiated and dormant antler stem cells (Dong et al. 2016). Notably, PI3K-AKT, never identified in red deer antler, played an indispensable role in sika deer antler regeneration. We found that red deer or sika deer was selected as experimental animal to study on antler regeneration in last decade. And both of them have the potential to be developed as the model of regenerative medicine. However, the difference of antler regeneration between two deer species still remains unknown. Unfortunately, previous study showed that the histogenetic aspects of these antlers were indistinguishable between red deer and sika deer ( $\mathrm{Li} 2013$; Li et al. 2014). Except for the size of two antler groups, we never know any other differences between the two antler groups before. In some cases, the term "antler" means that the antler collected from sika deer or red deer. The antler, as Chinese tradition medicine, is collected from sika deer or red deer according to Chinese Pharmacopeia (Pharmacopeia 2015). We realize that comparative study of antler regeneration between red deer and sika deer has been a "scotoma" all the time. Hence, it is necessary to make the comparative study of these two antler groups. In our current study, the proteomics technology was applied to reveal the differences of the protein level between them.

The aim of our study was to disclose the differentially expressed proteins between two antler groups. Furthermore, we want to remind other researchers that these two antler systems are different. The researchers should notice that whether these different pathways affect their study, when they choose antler to study on regenerative biomedicine. The proteomics based on mass spectrum can identify thousands of proteins, which were processed by bioinformatic software to achieve the protein properties, consisting of protein abundance, protein subcellular distribution, protein functional classification, and correlative biological processes (Larance \& Lamond 2015). Thus, proteomics will accurately work out essentially changed molecular pathways of antler regeneration between red deer and sika deer. The differentially expressed proteins identified in comparative proteomic may facilitate the discovery of novel molecular pathways related with

We randomly choose three male red deer and three male sika deer as study subjects in Chinese local deer farm, which was located in Shuangyang district, Changchun city, Jilin province $\left(\mathrm{N} 43^{\circ} 33^{\prime} 56.03^{\prime \prime}, \mathrm{E} 125^{\circ} 29^{\prime} 23.15^{\prime \prime}\right)$. All procedures of the sample collection were approved by the Animal Ethics Committee of Changchun University of Chinese Medicine. They are all 5-yearold deer with antler regeneration for 80 days after casting previous antlers. Antler tissues were 
94 harvested from anaesthetized deer. Briefly, the distal $5 \mathrm{~cm}$ of the antler tip, regarded as growth

95 center, was removed and sectioned sagittally by the electric saw to collect $5 \mathrm{~mm}$ to $8 \mathrm{~mm}$ thick

96 tissue section ( $\mathrm{Li}$ et al. 2005). And then tissue section was cut into small pieces, flash frozen and

97 ground into powder in liquid nitrogen, combined and stored at $-80{ }^{\circ} \mathrm{C}$ ready for proteomics

98 study.

99

100

101

102

103

\section{Protein extraction and FASP digestion}

The antler powder samples were extracted with SDT-lysis buffer (4\% (w/v) SDS, 100mM Tris$\mathrm{HCl} \mathrm{pH} \mathrm{7.6,} \mathrm{0.1M} \mathrm{DTT)} \mathrm{in} \mathrm{a} \mathrm{sonic} \mathrm{dismembrator} \mathrm{for} 20 \mathrm{~min}$. And then the suspension was incubated at $4{ }^{\circ} \mathrm{C}$ for $60 \mathrm{~min}$ and subsequently heated at $95^{\circ} \mathrm{C}$ for $5 \mathrm{~min}$. The incubation solution was centrifuged at $16000 \mathrm{~g}$ at $4{ }^{\circ} \mathrm{C}$ for $15 \mathrm{~min}$. The clarified supernatant was collected to another tube, and then the pellet extracted again in lysis buffer according to the above method. The

106

107

108

109

110

111

112

113

114

115

116

117

118

119

120

121

122

123

124

125

126

127

128

129

130

131

132

133

combination of supernatants was centrifuged at $30000 \mathrm{~g}$ and $4{ }^{\circ} \mathrm{C}$ for $15 \mathrm{~min}$. The concentration of proteins was quantified using the bicinchoninic acid assay (BCA assay). $200 \mu \mathrm{g}$ of protein from each antler sample was digested with trypsin according to filter-aided sample preparation (FASP) protocol proposed by Wisniewski et al. (Wisniewski et al. 2009). Subsequently, $2 \mu \mathrm{g}$ of tryptic peptides were purified using $\mathrm{C} 18$ spin columns (Pierce ${ }^{\mathrm{TM}} \mathrm{C} 18$, Sigma), and then dried via lyophilization and rehydrated with $40 \mu \mathrm{l}$ of $0.1 \%$ formic acid. The content of the peptide sample was quantified based on colorimetric protein concentration assays (BioRad).

\section{LC-MS/MS analysis}

Purified digested peptide fractions were analyzed by nano LC-MS/MS using EASY-nLC 1200 coupled to Q Exactive ${ }^{\mathrm{TM}}$ mass spectrometer (Thermo Fisher Scientific). The peptide samples were separated with a 120 min linear gradient from $0 \%$ to $55 \%$ buffer B ( $84 \%$ acetonitrile, $0.1 \%$ Formic acid) on a reverse phase peptide column (Acclaim ${ }^{\mathrm{TM}}$ PepMap $^{\mathrm{TM}} 100 \mathrm{C} 18$, Thermo Fisher Scientific connected) to the EASY capillary C18 column $(75 \mu \mathrm{m}$ inner diameter, $10 \mathrm{~cm}$ long, $3 \mu \mathrm{m}$ resin, Thermo Fisher Scientific) at a flow rate of $300 \mathrm{nl} / \mathrm{min}$. The data-dependent top 10 method was used to acquire MS data by choosing the most abundant precursor ions for HCD fragmentation. The full MS survey scans were acquired at a resolution of 70,000 , with automatic gain control target $3 \mathrm{e} 6$ and a range of $\mathrm{m} / \mathrm{z} 300-1800$. HCD spectra scan with a resolution of $17,500 \mathrm{at} \mathrm{m} / \mathrm{z} 200$ and the isolation window was $2 \mathrm{~m} / \mathrm{z}$. The dynamic exclusion was $40 \mathrm{~s}$.

\section{Database search and protein quantification}

Mass spectra were processed by the peptide search engine Andromeda in MaxQuant software, which searched the data against uniprot_Cervus_20600_20180413.fasta retrieved from UniProt database. The correlated parameters were defined as follows: digest enzyme of trypsin, max missed cleavage of 2, fixed modifications of carbamidomethyl, variable modifications of methionine oxidation and $\mathrm{N}$-terminal acetylation, the precursor mass window in the main search of $6 \mathrm{ppm}$ and MS/MS tolerance of $20 \mathrm{ppm}$. The cutoff value of the false discovery rate was defined as 0.01 to identify peptides and proteins. The protein abundance was normalized and 
134 calculated according to the algorithms described in Cox et al (Tyanova et al. 2016a). Data from 135 the same antler group were merged into the same group, and the LFQ intensities of the proteins 136 were $\log 2$ transformed to obtain $\log 2$ fold-change between two antler groups. To improve the 137 accuracy of comparison, the data were retained with at least two of three valid values (greater 138 than 0) in at least one group, and missing values were replaced by generating random numbers 139 from a Gaussian distribution that well represents the distribution of low-abundance proteins 140 (Qian et al. 2018). If a certain protein has two valid values in one group and only one in the other 141 group, it will also be eliminated. Two-sample T-test is applied for determining if the means of 142 the LFQ intensity values of two groups of deer antlers are significantly different from each other

143 through the analysis of Perseus software (Tyanova et al. 2016b). To define differentially expressed proteins (DEPs), fold change thresholds were set at 2.0 or 0.5 with $p$-value $<0.05$. The mass spectrometry data in proteomics study have been submitted to the ProteomeXchange Consortium via PRIDE partner repository, with the dataset identifier PXD012090 (Vizcaino et al.

147 2016).

148

\section{Bioinformatic analysis}

150

151

To study on the biological function of the differentially expressed proteins, the identified proteins were used to run Gene Ontology (GO) analysis. Firstly, the homology search was

152

153

154

155

156

157

158

159

160

161

162

163

164

165

166

167

168

169

170

171

172

173 performed for all identified sequences against the red deer database using localized NCBI BLAST + software. And then GO annotation and enrichment analysis was completed using BLAST2GO software (Gotz et al. 2008). The result of GO annotation presented that the properties of the identified proteins in three functional categories, which are cellular component (CC), biological process (BP), and molecular function (MF). In addition, all differentially expressed proteins and significantly changed metabolites were searched against the Kyoto Encyclopedia of Genes and Genomes (KEGG) database and obtained the corresponding KEGG pathways (Moriya et al. 2007). To further disclose the mechanism underlying differentially expressed proteins and clarify the functional cluster of differentially expressed proteins, GO enrichment analysis was performed by the Fisher's exact test. Notably, GO terms with $p$ values $<0.05$ were regarded as significant enrichment.

\section{Results}

\section{Protein identification and differentially expressed proteins (DEPs)}

In total, 4439 unique peptides and 1060 proteins were identified through MaxQuant software against UniProt database. All identified protein from these two groups are listed in Table S1. An obvious di $\square$ erence in the number of proteins with changed abundance comparing red deer antler and sika deer antler make us to analyze the extent of shared and non-overlapping di $\square$ erences. 578 proteins were identified in both the red deer antler proteome and the sika deer antler proteome, while 114 proteins were exclusively identified in sika deer antler (Figure 1, Table S2). However, we found that only 19 exclusive proteins for red deer antler were observed (Figure 1, 
174 Table S2). Among these antler proteins existed in both red deer and sika deer, 30 differentially

175 expressed proteins (DEPs) were up-regulated and 17 down-regulated in the sika deer antler

176 (Figure 1, Table S2). Our current proteomics data indicated that more type of proteins were

177 identified in sika deer antler than that in red deer.

178

179

Gene ontology annotation and enrichment analysis of differentially expressed proteins

180

181

182

183

184

185

186

187

188

189

190

191

192

193

194

195

196

197

198

199

200

201

202

203

204

205

206

207

208

209

210

211

212

213

To further study protein function in deer antler, Gene ontology (GO) annotation was processed to differentially expressed proteins by Blast2GO software. All terms were counted non-exclusively, when one protein possessed more than one term for biological process (BP), molecular function (MF), and cellular component (CC). The result of GO annotation was shown in Figure 2 and Table S3. The most abundant category in molecular function was binding proteins (38\%), especially ion bonding proteins $(21 \%)$ and organic cyclic compound binding proteins $(20 \%)$ (Figure 2). The second highly abundant category in molecular function was catalytic activity proteins $(23 \%)$, especially hydrolase activity proteins $(10 \%)$. The cellular component analysis showed that the differentially expressed proteins were mainly located in the intracellular region, organelle and membrane (Figure 2). 23 and 18\% of proteins are in the organelle and membrane, respectively, whereas $9 \%$ of differentially expressed proteins are localized in the extracellular region. The major functional terms under biological process category were metabolic processes, cellular processes and biological regulation $(18,27$, and 11\%, respectively) (Figure 2). The nitrogen compound metabolic process, which is a child term of metabolic process, possessed 22 differentially expressed proteins from two antler groups (Table S3).

To reveal the biological difference between sika deer and red deer antler, GO enrichment analysis was performed for differentially expressed proteins on three categories (Figure 3). We found that differentially expressed proteins were mainly located in clathrin-coated pit/vesicles, organelle, specially mitochondrion and cytoskeleton. On the molecular function category, a high abundance of DEPs was enriched into transporter activity and ATPase activity. And DEPs were mainly involved in the regulation of gene expression and extracellular structure organization under biological process.

\section{KEGG annotation of differentially expressed proteins}

KEGG analysis was used to reveal the functional pathways about differentially expressed proteins. 180 DEPs were processed to obtain 163 KEGG pathways (Table S4). The top 20 pathway terms were shown in Figure 4. These differentially expressed proteins were mainly classified into PI3K-Akt signaling pathway, thermogenesis, oxidative phosphorylation, ECM-receptor interaction and focal adhesion, ribosome, synaptic vesicle cycle, complement and coagulation cascades, and other categories related with disease.

\section{Discussion}

\section{Proteins involved in oxidative phosphorylation}

During antler regeneration stage, mesenchymal cells (MSCs) differentiate into chondrocytes

Peer] reviewing PDF | (2018:03:26478:2:0:NEW 4 Jun 2019) 
214 participated in endochondral ossification, is one of the two key processes during bone

215 development (Rucklidge et al. 1997). Previous study has shown that throughout the osteogenesis

216 of MSCs, the copy number of mitochondrial DNA, oxygen consumption rate, OxPhos enzymes,

217 mRNA associated with mitochondrial biogenesis, and intracellular ATP content were increased

218 along with the significant decrease of endogenous reactive oxygen species (Chen et al. 2008;

219 Pattappa et al. 2011). The production of ATP is mainly driven by OxPhos enzymes located in

220 electron transport chain, where the proton gradient is built across the inner membrane by NADH-

221 coenzyme Q oxidoreductase (complex I), Q-cytochrome c oxidoreductase (complex III), and

222 cytochrome c oxidase (complex IV), which can drive ATP synthesis through ATP synthase

223 (complex V) (DiMauro \& Schon 2003).

224 According to KEGG annotation analysis, six up-regulated proteins in sika deer antler

225 participated in oxidative phosphorylation (OxPhos) (Table 1). Of these 6 up-regulated proteins,

226 vacuolar-type H-ATPase subunit E (ATP6E) belong to superfamily of related ATP synthases.

227 Co-expression of ATP8 and ATP6 genes provided stable protein expression for complex V

228 assemble of the oxidative phosphorylation complexes (Boominathan et al. 2016). The up-

229 regulation of V-ATPase gene (ATP6V0D2) was found in the zone of hypertrophic chondrocytes,

230 which was part of the process of endochondral ossification (Ayodele et al. 2017). The antlers

231 were regenerated through a modified endochondral ossification process that involves the

232 remodeling of cartilage (Faucheux et al. 2001). In this case, ATP6 could be the key enzyme

233 involved in ATP production during antler development, whereas ATP8 may take over the roles

234 of ATP6 in red deer. In addition, ATP8, which plays important roles in lipogenesis, was found

235 during the stage of porcine adipose tissue development (Zhang et al. 2016). Weiss et al. reported

236 that ATP8 mutation led to mitochondrial ROS generation with dysregulation of mitochondrial

237 OxPhos system (Weiss et al. 2012). It indicated that the high abundance of ATP8 in red deer

238 might maintain the stable of mitochondrial OxPhos system when antler regeneration faced an

239 increasing demand for energy. Moreover, cytochrome c oxidase subunit 6a (COX6A) and

240 cytochrome c oxidase subunit $6 \mathrm{~b}(\mathrm{COX} 6 \mathrm{~B})$ were significantly up-regulated in sika deer (Table

241 1). The enzyme cytochrome c oxidase (COX, also called complex IV), that consist of 14

242 subunits, is part of mitochondrial respiration oxidase (Balsa et al. 2012; Castresana et al. 1994).

243 The three key subunits of COX are synthesized in mitochondrial to form the functional core of

244 the enzyme, which is surrounded by 11 small nuclear-coded subunits (Balsa et al. 2012). The

245 activity of cytochrome c oxidase, complex IV of the mitochondrial respiratory chain, has been

246 shown to regulate the activity of osteoclasts necessary for adaptive bone remodeling (Gandhi et

247 al. 2017; Miyazaki et al. 2003). The disruption of osteoclast COX activity could lead to the loss

248 of bone-resorbing activity. The high expression of cytochrome c oxidase, accompanied with the

249 up-regulation of ATP6 in sika deer, indicated that antler remodeling is more active than red deer.

250 Accordingly, the higher ATP demand should be required for sika deer antler regeneration.

251 Previous studies have found that the overexpression of UQCRC1 enhanced complex III activity

252 in mice, and its down-regulation was related with significant dysfunction in mitochondrion of

253 epithelial cells (Kriaucionis et al. 2006; Shibanuma et al. 2011). This finding indicated that the

254 up-regulation of UQCRC1 in sika deer (Table 1) contributes to maintain normal mitochondrial

255 homeostasis, which is essential for osteoblast mediated bone formation (Pan et al. 2018). Etzler

256 et al. have reported that the up-regulation of Complex III in HEK293 cells (human embryonic

257 kidney cells) could enhance electron transport efficiency to survive from oxidative stress (Etzler

258 et al. 2017). And it was demonstrated that ROS inhibited the proliferation of chondrocytes and

259 induced the chondrocyte hypertrophy (Morita et al. 2007). Therefore, the current proteomic

Peer] reviewing PDF | (2018:03:26478:2:0:NEW 4 Jun 2019) 
260

261

262

263

264

265

266

267

268

269

270

271

272

273

274

275

276

277

278

279

280

281

282

283

284

285

286

287

288

289

290

291

292

293

294

295

296

297

298

299

300

301

302

303

304

305

study indicated that the antler development in sika deer require more complex III to regulate mitochondrial respiration in response to ROS, which was produced during endochondral ossification.

Recent work reported that the lower expression of NDUFS3 under oxidative stress impaired the Complex I assembly, which resulted in reduced Complex I content (Sen et al. 2015). Interestingly, the up-regulation of antioxidants, for example SOD2 and glutathione, could recover the complex I content (Gopal et al. 2018; Sen et al. 2015). According to the high abundance of NDUFS3, the content of antioxidants in sika deer antler should be higher that red deer (Table 1).

\section{The high abundance of large subunit ribosomal proteins in sika deer}

Another notable difference between red deer and sika deer antler was the higher abundance of large subunit ribosomal proteins (RPLs) in sika deer (Table 1). It is well known that ribosomes, as translational hub across the all living organisms, produce proteins based on mRNA template (de la Cruz et al. 2015; Khatter et al. 2015). The 80S ribosome in eukaryotes is comprised of a small (40S) and large (60S) subunit (Khatter et al. 2015). And the up-regulation of ribosomal proteins was necessary to meet protein synthesis requirements in the rapid growth stage of coho salmon (Causey et al. 2018). It is consistent with our result, where the higher abundance of ribosomal proteins in sika deer as compared to red deer could lead to synthesize a greater variety of proteins in sika deer (Figure S2). Therefore, ribosomal protein played a vital role in cell growth and proliferation by the regulation of protein biosynthesis. In bone marrow rRNAs, $60 \mathrm{~S}$ subunit biogenesis associated with cycle circle (Khincha et al. 2016; Ruggero \& Shimamura 2014). The mutation of RPL35A contributed to Diamond-Blackfan anemia, that is an inherited bone marrow failure syndrome (Khincha et al. 2016). Moreover, RPL4 was identified as a crucial regulator involved in MDM2-p53 pathway (He et al. 2016). RPL4 directly binds to MDM2 could significantly inhibit the ubiquitination and degradation of p53, resulting in the upregulation of p53. Interestingly, RPL5 and RPL11 also could bind to MDM2 in the same way for p53 induction under the assistant of RPL4 (Fumagalli et al. 2012; He et al. 2016; Sun et al. 2008). Further study had reported that the knockdown of large ribosome subunit proteins, RPL7a, RPL11, RPL5, RPL14, RPL26 and RPL35 would impair the ribosomal biogenesis (Bailly et al. 2016). The activation of p53 may promote endochondral ossification through interaction with proteins related with matrix mineralization and chondrocyte maturation or apoptosis (Li et al. 2012b). However, rpl3 regulated the cell cycle progression and apoptosis induction through p53-independent manner (Russo et al. 2016; Russo \& Russo 2017). The combination of the data suggested that high abundance of ribosomal protein large subunits in sika deer improved antler development, involving the process of endochondral ossification. Intriguingly, the content of ribosomal proteins in red deer was at relatively low level in contrast to sika deer.

\section{Difference of extracellular matrix proteins}

Type IX collagen (COL9) and cartilage oligomeric matrix protein (COMP), which are specifically expressed in cartilage tissue, participate in the regulation of cartilage development (Hecht et al. 2005; Myllyharju 2014). The skeletal deficiencies of mutations in COL9 or COMP strongly suggested that an adequate cartilage matrix was essential for extracellular matrix assembly and homeostasis (Myllyharju 2014; Shi et al. 2015). Mutations in COL9 or COMP have been shown to cause multiple epiphyseal dysplasia (MED), which was a rare genetic

Peer) reviewing PDF | (2018:03:26478:2:0:NEW 4 Jun 2019) 
306

307

308

309

310

311

312

313

314

315

316

317

318

319

320

321

322

323

324

325

326

327

328

329

330

331

332

333

334

335

336

337

338

339

340

341

342

343

344

345

346

347

348

349

350

351

disorder (Briggs \& Chapman 2002; Briggs et al. 1995). collagen IX knockout mouse model have implied that reduction of collagen IX changed the time course of callus differentiation during fracture repair (Opolka et al. 2007). The maturation of cartilage matrix was delayed in collagen IX knockout mice during the repair phase and the content of cartilage matrix was at relatively low concentration (Opolka et al. 2007). In addition, collagen IX will be gradually reduced as the mineralization of cartilage tissue. In the case of mutations in COMP, it reduced not only COMP secretion but type IX collagen in cartilage matrix (Hecht et al. 2005). In our antler proteomics study, the content of COL9 and COMP in sika deer was much higher than red deer (Table 1), which indicated that cartilage matrix assemble in sika deer was more active and red deer may possess more mineralized tissue zone. Consistently, laminin alpha 4 (LAMA4), that are the major constituent of hypertrophic chondrocytes, was highly expressed in sika deer (Table 1). It has reported that LAMA4 was implicated in chondrocyte mobility and chondrocyte hypertrophic zone (Fuerst et al. 2011; Moazedi-Fuerst et al. 2016). LAMA4 binding with integrins improved the chondrocyte mobility to promote the antler development, whilst the interaction between LAMA4 and integrins activated PI3K-AKT pathway to regulate the proliferation, differentiation and survival of chondrocytes. Osteopontin (OPN) is an extracellular matrix glycoprotein involved in bone remodeling (Denhardt \& Noda 1998). OPN was found to be secreted by different cell types as diverse as macrophages, epithelial cells, and osteoclasts (Rittling 2011; Tardelli et al. 2016). OPN, as migratory cytokine, stimulated the osteoclast migration to improve bone resorption. OPN is markedly expressed by MSCs and can be further up-regulated during the osteogenic differentiation (Chen et al. 2014; Rickard et al. 1994). OPN abundantly expressed in sika deer represented that the differentiation of MSCs putatively existed in the whole antler development stage. However, the abundance of OPN in red deer during late antler regeneration stage was significantly down-regulated, thereby retarded the mobility of osteoclast.

\section{PI3K-Akt pathway}

The Phosphoinositide 3-Kinase/Akt pathway is implicated in multiple cellular processes, including cell cycle entry, cell growth, cell survival and cell migration (Cantley 2002; Liu et al. 2009). A set of hormones, cytokines, and extracellular matrix (ECM) proteins, as stimulators, can activate PI3K/Akt pathway (Liu et al. 2009). Furthermore, activation of Akt signaling in cartilage development enhanced the proliferation of chondrocytes and suppressed the hypertrophic differentiation of chondrocytes through the down-regulation of Runx 2 expression, which participated in chondrocyte terminal differentiation (Kita et al. 2008). Compared with red deer, four ECM proteins in sika deer antler were significantly up-regulated (Table 1). Thereby, ECM proteins interacting with integrins can activate PI3K/Akt pathway through the action of focal adhesion kinase (FAK) in the sika deer. Meanwhile, the high abundance of guanine nucleotide-binding protein subunit gamma-12 (GNG12) in sika deer could can improve antler regeneration by activating PI3K (Table 1) (Luo et al. 2018). Another up-regulated protein belonging to PI3K/Akt pathway in sika deer, heat shock protein 90 (HSP90A), may contribute to mesenchymal stem cell migration to facilitate the antler development (Table 1) (Gao et al. 2015). Conversely, the up-regulation of serine/threonine protein phosphatase 2A (PP2A) in red deer will inhibit the dephosphorylate of Akt to inhibit PI3K/Akt pathway (Table 1) (Eichhorn et al. 2009).

\section{Conclusions}

Our comparative proteomics study provided the comprehensive analysis of the differentially expressed antler proteins between sika deer and red deer based on label-free quantitative 
352

353

354

355

356

357

358

359

360

361

362

363

364

365

366

367

368

369

370

371

372

373

374

375

376

377

378

379

380

381

382

383

384

385

386

387

388

389

390

391

392

393

394

395

396

397

398

proteomics. The protein species and their abundance of sika deer antler were higher than red deer. Differentially expressed proteins during antler regeneration stage mainly participated in the regulation of several pathways including oxidative phosphorylation, ribosome, extracellular matrix interaction, and PI3K-Akt pathway.

\section{References}

Ayodele BA, Mirams M, Pagel CN, and Mackie EJ. 2017. The vacuolar H(+) ATPase V0 subunit d2 is associated with chondrocyte hypertrophy and supports chondrocyte differentiation. Bone Rep 7:98-107. 10.1016/j.bonr.2017.08.002

Bailly A, Perrin A, Bou Malhab LJ, Pion E, Larance M, Nagala M, Smith P, O'Donohue MF, Gleizes PE, Zomerdijk J, Lamond Al, and Xirodimas DP. 2016. The NEDD8 inhibitor MLN4924 increases the size of the nucleolus and activates p53 through the ribosomalMdm2 pathway. Oncogene 35:415-426. 10.1038/onc.2015.104

Balsa E, Marco R, Pereles-Clemente E, Szklarczyk R, Calvo E, Landazuri MO, and Enriquez JA. 2012. NDUFA4 Is a Subunit of Complex IV of the Mammalian Electron Transport Chain. Cell Metabolism 16:378-386. 10.1016/j.cmet.2012.07.015

Banks WJ, and Newbrey JW. 1983. Antlers development as unique modification of mammalian endochondral ossification.

Boominathan A, Vanhoozer S, Basisty N, Powers K, Crampton AL, Wang X, Friedricks N, Schilling B, Brand MD, and O'Connor MS. 2016. Stable nuclear expression of ATP8 and ATP6 genes rescues a mtDNA Complex V null mutant. Nucleic Acids Res 44:93429357. 10.1093/nar/gkw756

Borsy A, Podani J, Steger V, Balla B, Horvath A, Kosa JP, Gyurjan I, Molnar A, Szabolcsi Z, Szabo L, Jako E, Zomborszky Z, Nagy J, Semsey S, Vellai T, Lakatos P, and Orosz L. 2009. Identifying novel genes involved in both deer physiological and human pathological osteoporosis. Molecular Genetics and Genomics 281:301-313. 10.1007/s00438-008-0413-7

Briggs MD, and Chapman KL. 2002. Pseudoachondroplasia and multiple epiphyseal dysplasia: mutation review, molecular interactions, and genotype to phenotype correlations. Hum Mutat 19:465-478. 10.1002/humu.10066

Briggs MD, Hoffman SM, King LM, Olsen AS, Mohrenweiser H, Leroy JG, Mortier GR, Rimoin DL, Lachman RS, and Gaines ES. 1995. Pseudoachondroplasia and multiple epiphyseal dysplasia due to mutations in the cartilage oligomeric matrix protein gene. Nat Genet 10:330-336. 10.1038/ng0795-330

Cantley LC. 2002. The phosphoinositide 3-kinase pathway. Science 296:1655-1657. 10.1126/science.296.5573.1655

Castresana J, Lubben M, Saraste M, and Higgins DG. 1994. Evolution of cytochrome oxidase, an enzyme older than atmospheric oxygen. EMBO J 13:2516-2525.

Causey DR, Kim JH, Stead DA, Martin SAM, Devlin RH, and Macqueen DJ. 2018. Proteomic comparison of selective breeding and growth hormone transgenesis in fish: Unique pathways to enhanced growth. J Proteomics. 10.1016/j.jprot.2018.08.013

Chen CT, Shih YR, Kuo TK, Lee OK, and Wei YH. 2008. Coordinated changes of mitochondrial biogenesis and antioxidant enzymes during osteogenic differentiation of human mesenchymal stem cells. Stem Cells 26:960-968. 10.1634/stemcells.2007-0509 
399

400

401

402

403

404

405

406

407

408

409

410

411

412

413

414

415

416

417

418

419

420

421

422

423

424

425

426

427

428

429

430

431

432

433

434

435

436

437

438

439

440

441

442

443

444

445

446

447

448

449

Chen Q, Shou P, Zhang L, Xu C, Zheng C, Han Y, Li W, Huang Y, Zhang X, Shao C, Roberts Al, Rabson AB, Ren G, Zhang Y, Wang Y, Denhardt DT, and Shi Y. 2014. An osteopontin-integrin interaction plays a critical role in directing adipogenesis and osteogenesis by mesenchymal stem cells. Stem Cells 32:327-337. 10.1002/stem.1567

de la Cruz J, Karbstein K, and Woolford JL, Jr. 2015. Functions of ribosomal proteins in assembly of eukaryotic ribosomes in vivo. Annu Rev Biochem 84:93-129. 10.1146/annurev-biochem-060614-033917

Deb-Choudhury S, Wang WY, Clerens S, McMahon C, Dyer JM, and Li CY. 2015. Direct localisation of molecules in tissue sections of growing antler tips using MALDI imaging. Molecular and Cellular Biochemistry 409:225-241. 10.1007/s11010-015-2527-7

Denhardt DT, and Noda M. 1998. Osteopontin expression and function: Role in bone remodeling. J Cell Biochem 72 Suppl 30-31:92-102. 10.1002/(SICI)10974644(1998)72:30/31+<92::AID-JCB13>3.0.CO;2-A

DiMauro S, and Schon EA. 2003. Mitochondrial respiratory-chain diseases. N Engl J Med 348:2656-2668. 10.1056/NEJMra022567

Dong Z, Ba H, Zhang W, Coates D, and Li C. 2016. iTRAQ-Based Quantitative Proteomic Analysis of the Potentiated and Dormant Antler Stem Cells. International Journal of Molecular Sciences 17. 10.3390/ijms 17111778

Eichhorn PJ, Creyghton MP, and Bernards R. 2009. Protein phosphatase 2A regulatory subunits and cancer. Biochim Biophys Acta 1795:1-15. 10.1016/j.bbcan.2008.05.005

Etzler JC, Bollo M, Holstein D, Deng JJ, Perez V, Lin DT, Richardson A, Bai Y, and Lechleiter JD. 2017. Cyclophilin D over-expression increases mitochondrial complex III activity and accelerates supercomplex formation. Arch Biochem Biophys 613:61-68. 10.1016/j.abb.2016.11.008

Faucheux C, Nesbitt SA, Horton MA, and Price JS. 2001. Cells in regenerating deer antler cartilage provide a microenvironment that supports osteoclast differentiation. J Exp Biol 204:443-455.

Fennessy PF. 1984. Deer Antlers: Regeneration, function and evolution. Journal of the Royal Society of New Zealand 14:290-291.

Fuerst FC, Gruber G, Stradner MH, Jones JC, Kremser ML, Angerer H, Setznagl D, Glehr M, Windhager R, Leithner A, and Graninger WB. 2011. Regulation of MMP3 by laminin alpha 4 in human osteoarthritic cartilage. Scand $J$ Rheumatol 40:494-496. 10.3109/03009742.2011.605392

Fumagalli S, Ivanenkov VV, Teng T, and Thomas G. 2012. Suprainduction of p53 by disruption of $40 S$ and $60 S$ ribosome biogenesis leads to the activation of a novel G2/M checkpoint. Genes Dev 26:1028-1040. 10.1101/gad.189951.112

Gandhi SS, Muraresku C, McCormick EM, Falk MJ, and McCormack SE. 2017. Risk factors for poor bone health in primary mitochondrial disease. J Inherit Metab Dis 40:673-683. 10.1007/s10545-017-0046-2

Gao F, Hu X, Xie X, Liu X, and Wang J. 2015. Heat shock protein 90 stimulates rat mesenchymal stem cell migration via PI3K/Akt and ERK1/2 pathways. Cell Biochem Biophys 71:481-489. 10.1007/s12013-014-0228-6

Gopal RK, Calvo SE, Shih AR, Chaves FL, McGuone D, Mick E, Pierce KA, Li Y, Garofalo A, Van Allen EM, Clish CB, Oliva E, and Mootha VK. 2018. Early loss of mitochondrial complex I and rewiring of glutathione metabolism in renal oncocytoma. Proc Natl Acad Sci U S A 115:E6283-E6290. 10.1073/pnas.1711888115

Gotz S, Garcia-Gomez JM, Terol J, Williams TD, Nagaraj SH, Nueda MJ, Robles M, Talon M, Dopazo J, and Conesa A. 2008. High-throughput functional annotation and data mining with the Blast2GO suite. Nucleic Acids Res 36:3420-3435. 10.1093/nar/gkn176

Gyurjan I, Molnar A, Borsy A, Steger V, Hackler L, Zomborszky Z, Papp P, Duda E, Deak F, Lakatos P, Puskas LG, and Orosz L. 2007. Gene expression dynamics in deer antler:

PeerJ reviewing PDF | (2018:03:26478:2:0:NEW 4 Jun 2019) 
450

451

452

453

454

455

456

457

458

459

460

461

462

463

464

465

466

467

468

469

470

471

472

473

474

475

476

477

478

479

480

481

482

483

484

485

486

487

488

489

490

491

492

493

494

495

496

497

498

499

mesenchymal differentiation toward chondrogenesis. Molecular Genetics and Genomics 277:221-235. 10.1007/s00438-006-0190-0

He X, Li YH, Dai MS, and Sun XX. 2016. Ribosomal protein L4 is a novel regulator of the MDM2-p53 loop. Oncotarget 7:16217-16226. DOI 10.18632/oncotarget.7479

Hecht JT, Hayes E, Haynes R, and Cole WG. 2005. COMP mutations, chondrocyte function and cartilage matrix. Matrix Biology 23:525-533. 10.1016/j.matbio.2004.09.006

Hu P, Wang T, Liu H, Xu J, Wang L, Zhao P, and Xing X. 2019. Full-length transcriptome and microRNA sequencing reveal the specific gene-regulation network of velvet antler in sika deer with extremely different velvet antler weight. Molecular Genetics and Genomics 294:431-443. 10.1007/s00438-018-1520-8

Khatter H, Myasnikov AG, Natchiar SK, and Klaholz BP. 2015. Structure of the human 80 S ribosome. Nature 520:640-645. 10.1038/nature14427

Khincha P, Mirabello L, Ellis SR, Giri N, Brodie S, Chandrasekharappa S, Donovan F, Zhou WY, Hicks B, Boland J, Yeager M, Jones K, Zhu B, Wang MY, Alter BP, and Savage SA. 2016. Novel and Known Ribosomal Causes of Diamond-Blackfan Anemia Identified through Comprehensive Genomic Characterization. Blood 128.

Kita K, Kimura T, Nakamura N, Yoshikawa H, and Nakano T. 2008. PI3K/Akt signaling as a key regulatory pathway for chondrocyte terminal differentiation. Genes to Cells 13:839-850. 10.1111/j.1365-2443.2008.01209.x

Kriaucionis S, Paterson A, Curtis J, Guy J, Macleod N, and Bird A. 2006. Gene expression analysis exposes mitochondrial abnormalities in a mouse model of Rett syndrome. Mol Cell Biol 26:5033-5042. 10.1128/MCB.01665-05

Larance M, and Lamond Al. 2015. Multidimensional proteomics for cell biology. Nat Rev Mol Cell Biol 16:269-280. 10.1038/nrm3970

Li C. 2013. Histogenetic aspects of deer antler development. Front Biosci (Elite Ed) 5:479-489.

Li C, Mackintosh CG, Martin SK, and Clark DE. 2007. Identification of key tissue type for antler regeneration through pedicle periosteum deletion. Cell \& Tissue Research 328:65-75.

Li C, and Suttie JM. 1994. Light microscopic studies of pedicle and early first antler development in red deer (Cervus elaphus). Anatomical Record-advances in Integrative Anatomy \& Evolutionary Biology 239:198-215.

Li C, Suttie JM, and Clark DE. 2004. Morphological observation of antler regeneration in red deer (Cervus elaphus). Journal of Morphology 262:731.

Li C, Suttie JM, and Clark DE. 2005. Histological examination of antler regeneration in red deer (Cervus elaphus). Anat Rec A Discov Mol Cell Evol Biol 282:163-174. 10.1002/ar.a.20148

Li C, Zhao H, Liu Z, and McMahon C. 2014. Deer antler--a novel model for studying organ regeneration in mammals. Int J Biochem Cell Biol 56:111-122. 10.1016/j.biocel.2014.07.007

Li CY, Harper A, Puddick J, Wang WY, and McMahon C. 2012a. Proteomes and Signalling Pathways of Antler Stem Cells. Plos One 7. ARTN e30026 10.1371/journal.pone.0030026

Li F, Lu Y, Ding M, Wu G, Sinha S, Wang S, and Zheng Q. 2012b. Putative function of TAP63alpha during endochondral bone formation. Gene 495:95-103. 10.1016/j.gene.2011.12.057

Liu P, Cheng H, Roberts TM, and Zhao JJ. 2009. Targeting the phosphoinositide 3-kinase pathway in cancer. Nat Rev Drug Discov 8:627-644. 10.1038/nrd2926

Luo C, Zhao S, Dai W, Zheng N, and Wang J. 2018. Proteomic analyses reveal GNG12 regulates cell growth and casein synthesis by activating the Leu-mediated mTORC1 signaling pathway. Biochim Biophys Acta Proteins Proteom 1866:1092-1101. 10.1016/j.bbapap.2018.08.013

PeerJ reviewing PDF | (2018:03:26478:2:0:NEW 4 Jun 2019) 
500

501

502

503

504

505

506

507

508

509

510

511

512

513

514

515

516

517

518

519

520

521

522

523

524

525

526

527

528

529

530

531

532

533

534

535

536

537

538

539

540

541

542

543

544

545

546

547

548

549

550

Miyazaki T, Neff L, Tanaka S, Horne WC, and Baron R. 2003. Regulation of cytochrome c oxidase activity by c-Src in osteoclasts. J Cell Biol 160:709-718. 10.1083/jcb.200209098

Moazedi-Fuerst FC, Gruber G, Stradner MH, Guidolin D, Jones JC, Bodo K, Wagner K, Peischler D, Krischan V, Weber J, Sadoghi P, Glehr M, Leithner A, and Graninger WB. 2016. Effect of Laminin-A4 inhibition on cluster formation of human osteoarthritic chondrocytes. J Orthop Res 34:419-426. 10.1002/jor.23036

Molnar A, Gyurjan I, Korpos E, Borsy A, Steger V, Buzas Z, Kiss I, Zomborszky Z, Papp P, Deak F, and Orosz L. 2007. Identification of differentially expressed genes in the developing antler of red deer Cervus elaphus. Molecular Genetics and Genomics 277:237-248. 10.1007/s00438-006-0193-x

Morita K, Miyamoto T, Fujita N, Kubota Y, Ito K, Takubo K, Miyamoto K, Ninomiya K, Suzuki T, Iwasaki R, Yagi M, Takaishi H, Toyama Y, and Suda T. 2007. Reactive oxygen species induce chondrocyte hypertrophy in endochondral ossification. J Exp Med 204:16131623. 10.1084/jem.20062525

Moriya Y, Itoh M, Okuda S, Yoshizawa AC, and Kanehisa M. 2007. KAAS: an automatic genome annotation and pathway reconstruction server. Nucleic Acids Res 35:W182185. 10.1093/nar/gkm321

Myllyharju J. 2014. Extracellular Matrix and Developing Growth Plate. Current Osteoporosis Reports 12:439-445. 10.1007/s11914-014-0232-1

Opolka A, Ratzinger S, Schubert T, Spiegel HU, Grifka J, Bruckner P, Probst A, and Grassel S. 2007. Collagen IX is indispensable for timely maturation of cartilage during fracture repair in mice. Matrix Biology 26:85-95. 10.1016/j.matbio.2006.09.010

Pan JX, Tang F, Xiong F, Xiong L, Zeng P, Wang B, Zhao K, Guo H, Shun C, Xia WF, Mei L, and Xiong WC. 2018. APP promotes osteoblast survival and bone formation by regulating mitochondrial function and preventing oxidative stress. Cell Death Dis 9:1077. 10.1038/s41419-018-1123-7

Pattappa G, Heywood HK, de Bruijn JD, and Lee DA. 2011. The metabolism of human mesenchymal stem cells during proliferation and differentiation. J Cell Physiol 226:25622570. 10.1002/jcp.22605

Pharmacopeia NCo. 2015. Pharmacopeia of the Peopole's Republic of China. Beijing: China Medical Science Press.

Qian C, Chen HM, Johs A, Lu X, An J, Pierce EM, Parks JM, Elias DA, Hettich RL, and Gu BH. 2018. Quantitative Proteomic Analysis of Biological Processes and Responses of the Bacterium Desulfovibrio desulfuricans ND132 upon Deletion of Its Mercury Methylation Genes. Proteomics 18. ARTN 1700479

10.1002/pmic.201700479

Rickard DJ, Sullivan TA, Shenker BJ, Leboy PS, and Kazhdan I. 1994. Induction of rapid osteoblast differentiation in rat bone marrow stromal cell cultures by dexamethasone and BMP-2. Dev Biol 161:218-228. 10.1006/dbio.1994.1022

Rittling SR. 2011. Osteopontin in macrophage function. Expert Rev Mol Med 13:e15. 10.1017/S1462399411001839

Rucklidge GJ, Milne G, Bos KJ, Farquharson C, and Robins SP. 1997. Deer antler does not represent a typical endochondral growth system: immunoidentification of collagen type $X$ but little collagen type II in growing antler tissue. Comp Biochem Physiol B Biochem Mol Biol 118:303-308.

Ruggero D, and Shimamura A. 2014. Marrow failure: a window into ribosome biology. Blood 124:2784-2792. 10.1182/blood-2014-04-526301

Russo A, Pagliara V, Albano F, Esposito D, Sagar V, Loreni F, Irace C, Santamaria R, and Russo G. 2016. Regulatory role of rpL3 in cell response to nucleolar stress induced by Act $D$ in tumor cells lacking functional p53. Cell Cycle 15:41-51.

10.1080/15384101.2015.1120926

PeerJ reviewing PDF | (2018:03:26478:2:0:NEW 4 Jun 2019) 
551

552

553

554

555

556

557

558

559

560

561

562

563

564

565

566

567

568

569

570

571

572

573

574

575

576

577

578

579

580

581

582

583

584

585

586

587

588

589

590

591

592

593

594

595

596

597

598

Russo A, and Russo G. 2017. Ribosomal Proteins Control or Bypass p53 during Nucleolar Stress. International Journal of Molecular Sciences 18. ARTN 140

10.3390/ijms 18010140

Sen S, Domingues CC, Rouphael C, Chou C, Kim C, and Yadava N. 2015. Genetic modification of human mesenchymal stem cells helps to reduce adiposity and improve glucose tolerance in an obese diabetic mouse model. Stem Cell Res Ther 6:242. 10.1186/s13287-015-0224-9

Shi XW, Zhang F, Lv AL, Wen Y, and Guo X. 2015. COL9A1 Gene Polymorphism Is Associated with Kashin-Beck Disease in a Northwest Chinese Han Population. Plos One 10. ARTN e0120365

10.1371/journal.pone. 0120365

Shibanuma M, Inoue A, Ushida K, Uchida T, Ishikawa F, Mori K, and Nose K. 2011. Importance of mitochondrial dysfunction in oxidative stress response: A comparative study of gene expression profiles. Free Radic Res 45:672-680. 10.3109/10715762.2011.564169

Steger V, Molnar A, Borsy A, Gyurjan I, Szabolcsi Z, Dancs G, Molnar J, Papp P, Nagy J, Puskas L, Barta E, Zomborszky Z, Horn P, Podani J, Semsey S, Lakatos P, and Orosz L. 2010. Antler development and coupled osteoporosis in the skeleton of red deer Cervus elaphus: expression dynamics for regulatory and effector genes. Molecular Genetics and Genomics 284:273-287. 10.1007/s00438-010-0565-0

Sun XX, Dai MS, and Lu H. 2008. Mycophenolic acid activation of p53 requires ribosomal proteins L5 and L11. J Biol Chem 283:12387-12392. 10.1074/jbc.M801387200

Tardelli M, Zeyda K, Moreno-Viedma V, Wanko B, Grun NG, Staffler G, Zeyda M, and Stulnig TM. 2016. Osteopontin is a key player for local adipose tissue macrophage proliferation in obesity. Molecular Metabolism 5:1131-1137. 10.1016/j.molmet.2016.09.003

Tyanova S, Temu T, and Cox J. 2016a. The MaxQuant computational platform for mass spectrometry-based shotgun proteomics. Nature Protocols 11:2301-2319. 10.1038/nprot.2016.136

Tyanova S, Temu T, Sinitcyn P, Carlson A, Hein MY, Geiger T, Mann M, and Cox J. 2016b. The Perseus computational platform for comprehensive analysis of (prote)omics data. Nat Methods 13:731-740. 10.1038/nmeth.3901

Vizcaino JA, Csordas A, Del-Toro N, Dianes JA, Griss J, Lavidas I, Mayer G, Perez-Riverol Y, Reisinger F, Ternent T, Xu QW, Wang R, and Hermjakob H. 2016. 2016 update of the PRIDE database and its related tools. Nucleic Acids Res 44:11033. 10.1093/nar/gkw880

Weiss H, Wester-Rosenloef L, Koch C, Koch F, Baltrusch S, Tiedge M, and Ibrahim S. 2012. The mitochondrial Atp8 mutation induces mitochondrial ROS generation, secretory dysfunction, and beta-cell mass adaptation in conplastic B6-mtFVB mice. Endocrinology 153:4666-4676. 10.1210/en.2012-1296

Wisniewski JR, Zougman A, Nagaraj N, and Mann M. 2009. Universal sample preparation method for proteome analysis. Nat Methods 6:359-362. 10.1038/nmeth.1322

Yao BJ, Zhao Y, Wang Q, Zhang M, Liu MC, Liu HL, and Li J. 2012. De novo characterization of the antler tip of Chinese Sika deer transcriptome and analysis of gene expression related to rapid growth. Molecular and Cellular Biochemistry 364:93-100. 10.1007/s11010-011-1209-3

Zhang J, Ma J, Long K, Jin L, Liu Y, Zhou C, Tian S, Chen L, Luo Z, Tang Q, Jiang A, Wang X, Wang D, Jiang Z, Wang J, Li X, and Li M. 2016. Dynamic gene expression profiles during postnatal development of porcine subcutaneous adipose. PeerJ 4:e1768. 10.7717/peerj. 1768

PeerJ reviewing PDF | (2018:03:26478:2:0:NEW 4 Jun 2019) 


\section{Figure 1}

Venn diagram of identified proteins from sika deer and red deer groups.

The identified antler proteins in at least two of three replicates were shown in the diagram.

The overlapping regions indicate the number of shared proteins. The number above or below the horizontal line in each portion shows the number of up- and down-regulated shared proteins between sika deer group versus red deer group

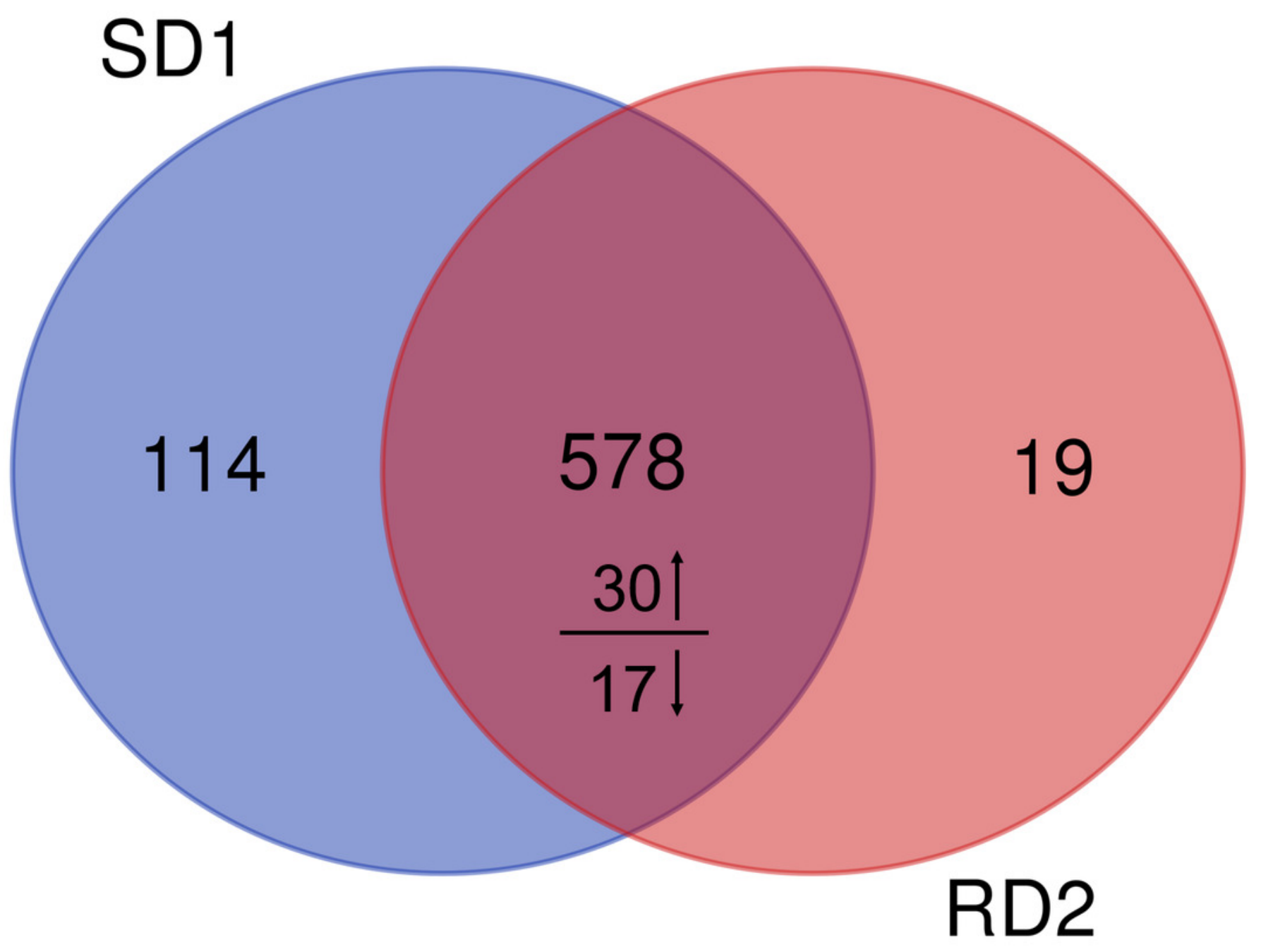


Figure 2

GO annotation for the differentially expressed antler proteins between sika deer and red deer.

The GO annotation results are displayed under three main categories: biological process, molecular function, and cellular component.

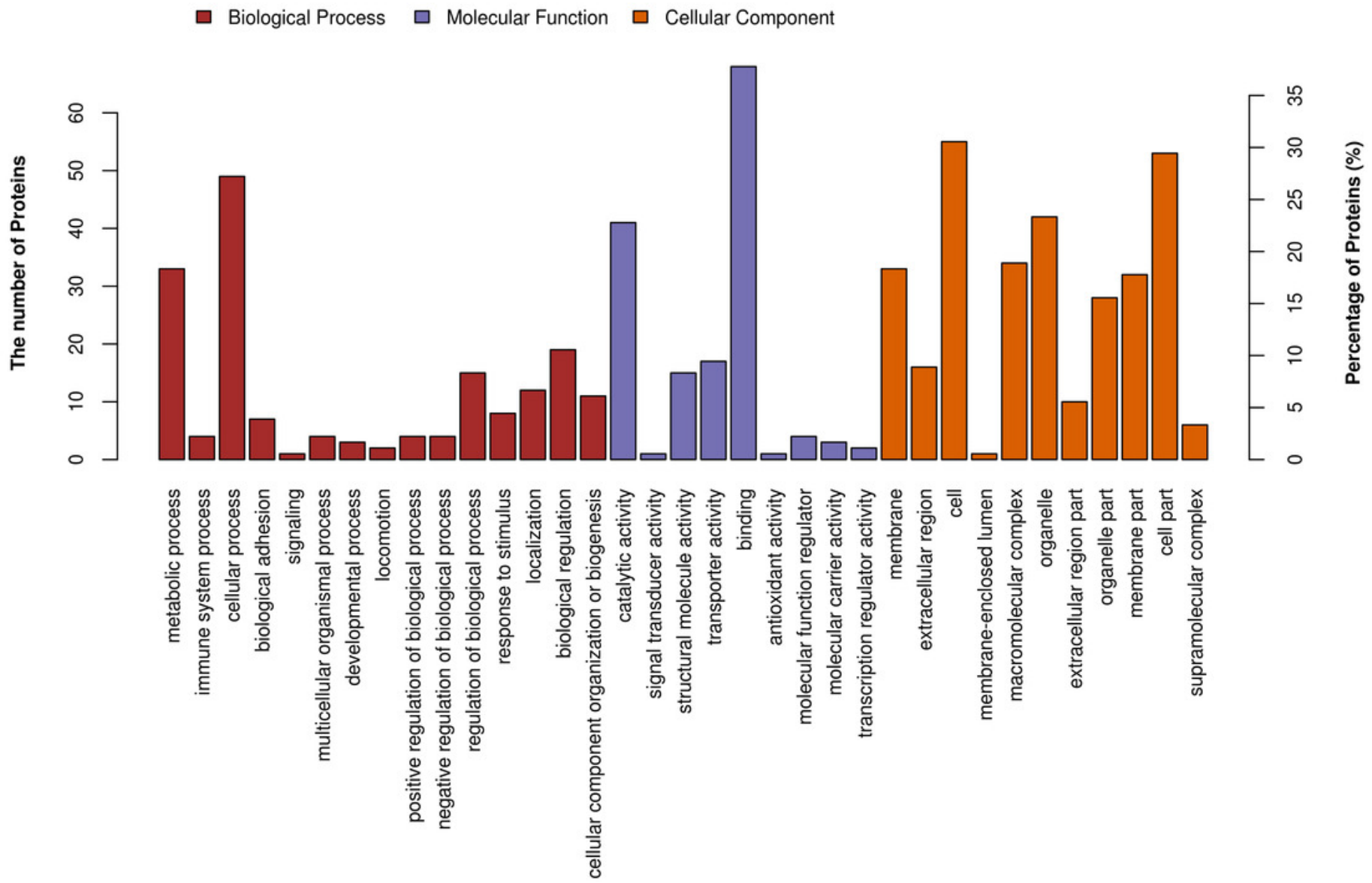




\section{Figure 3}

GO enrichment analysis for the differentially expressed antler proteins between sika deer and red deer.

The color of the bar chart represents the significance of the GO Enrichment. The color gradient corresponds to the magnitude of $\mathrm{P}$ value. The gradient becomes red, which indicates a low $P$ value. The value on the top of the bar chart is the rich factor (rich factor $\leq 1)$. The rich factor is the ratio of the number of differentially expressed proteins associated with a certain $\mathrm{GO}$ term to the number of all identified proteins associated with a certain GO term 


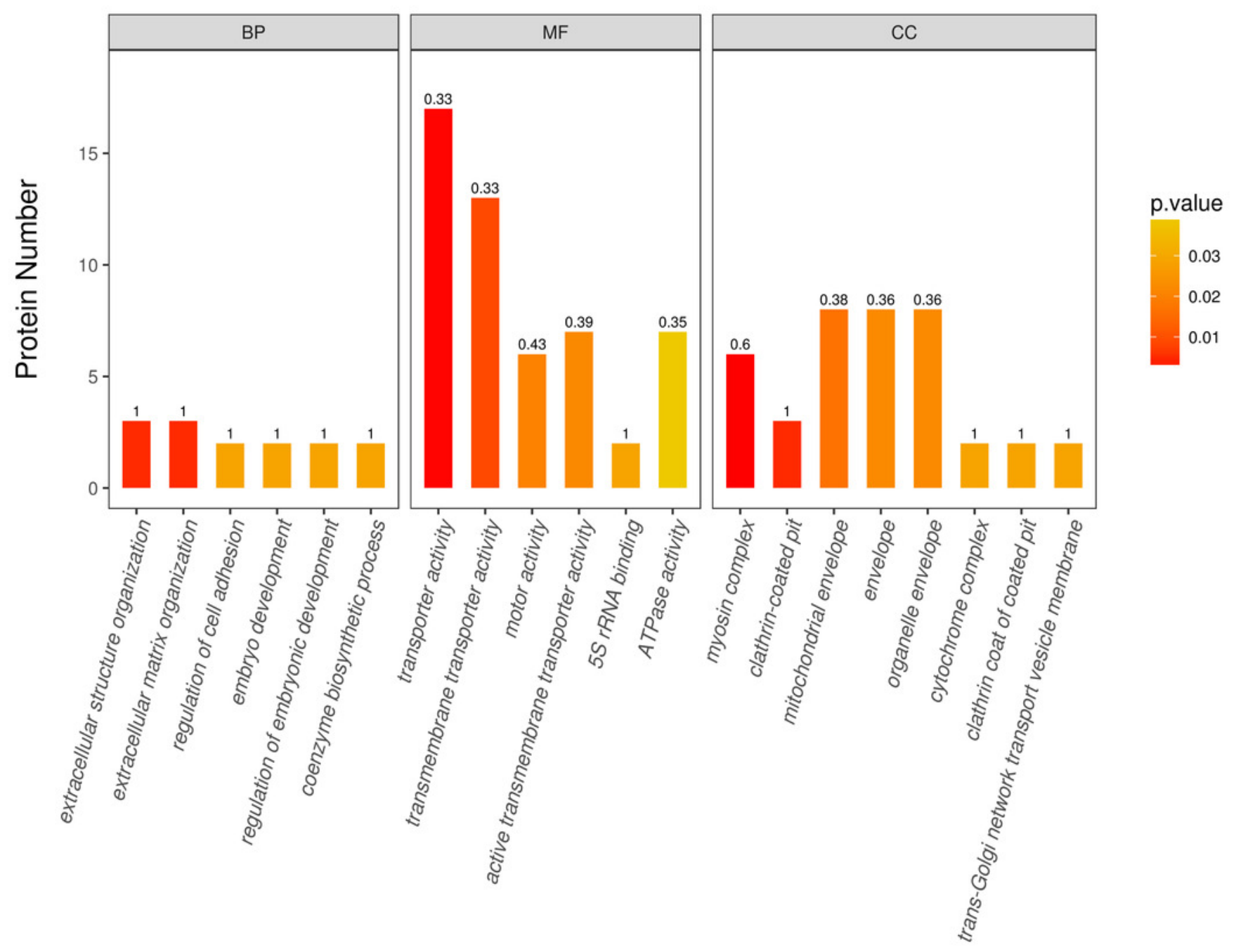

Enriched GO Terms (Top 20) 
Figure 4

KEGG annotation for the differentially expressed antler proteins between sika deer and red deer.

The top of the bar chart shows the number of identified proteins involved KEGG pathways.

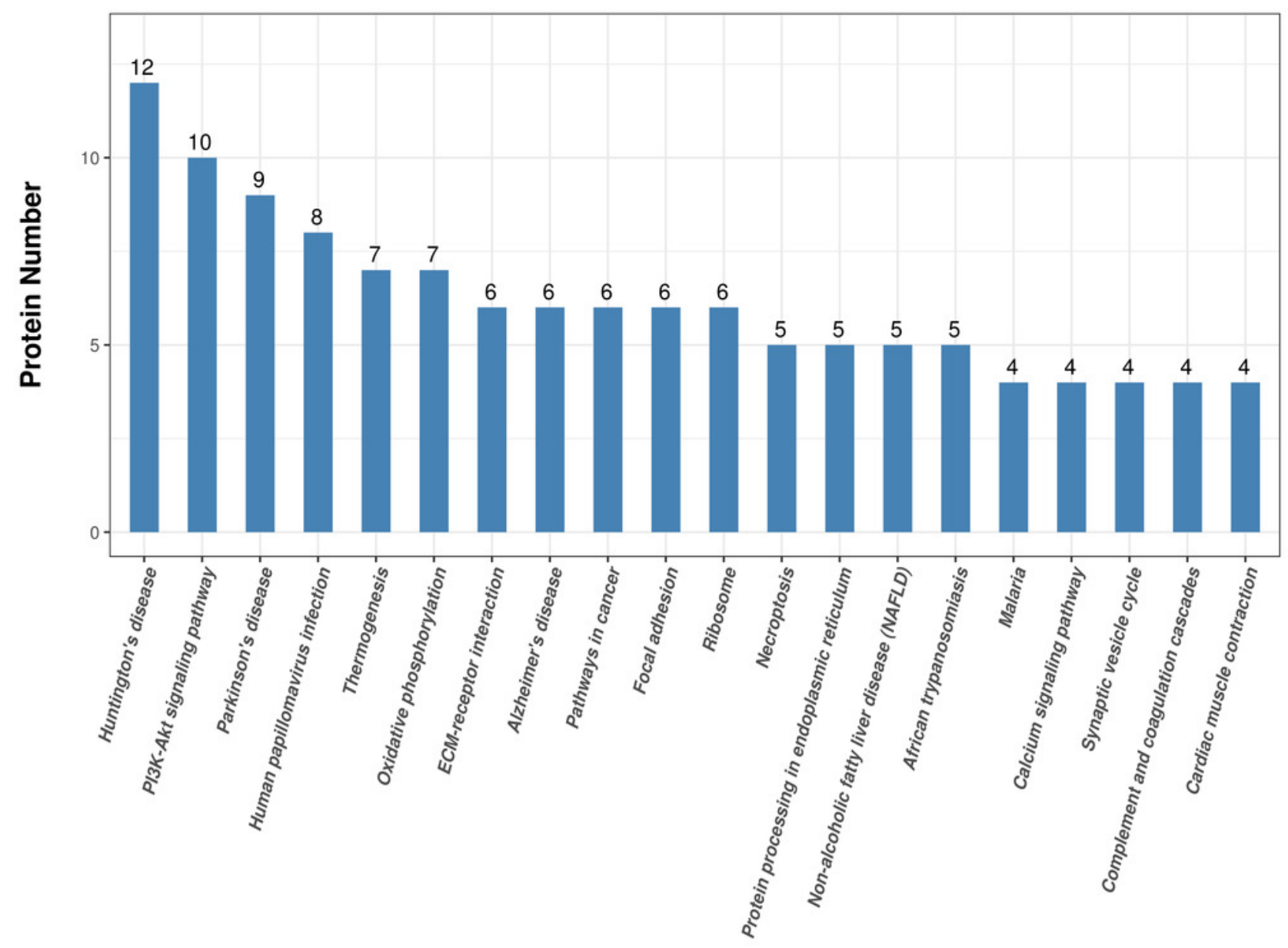

KEGG Pathways (Top 20) 


\section{Table 1 (on next page)}

Differentially expressed proteins involved in KEGG pathway. 
1 Table 1. Differentially expressed proteins involved in KEGG pathways.

\begin{tabular}{llll}
\hline Oxidative Phosphorylation Pathway & & \\
\hline UniProt ID & Protein Name & RD2/SD1 & P-value \\
\hline A0A172ZQ52 & ATP8 & 2.232388 & 0.018918192 \\
\hline A0A212CA05 & QCR1, UQCRC1 & SD1 ${ }^{1}$ & - \\
\hline A0A212CD21 & ATPeV1E, ATP6E & SD1 ${ }^{1}$ & - \\
\hline A0A212CE28 & COX6C & SD1 ${ }^{1}$ & - \\
\hline A0A212D8C1 & COX6A & SD1 ${ }^{1}$ & - \\
\hline A0A212D8Y9 & QCR9, UCRC & SD1 ${ }^{1}$ & - \\
\hline A0A212DI17 & NDUFS3 & SD1 ${ }^{1}$ & - \\
\hline
\end{tabular}

\begin{tabular}{|c|c|c|c|}
\hline \multicolumn{4}{|c|}{ Ribosome Pathway } \\
\hline UniProt ID & Protein Name & RD2/SD1 & $P$-value \\
\hline A0A212CGQ3 & large subunit ribosomal protein $\mathrm{L} 14 \mathrm{e}$ & 0.466 & 0.00337 \\
\hline A0A212CIR2 & large subunit ribosomal protein L35A & 0.429 & 0.0408 \\
\hline $\mathrm{A} 0 \mathrm{~A} 212 \mathrm{CD} 44$ & large subunit ribosomal protein L3e & SD1 ${ }^{1}$ & - \\
\hline $\mathrm{A} 0 \mathrm{~A} 212 \mathrm{CH} 34$ & large subunit ribosomal protein L5e & SD1 ${ }^{1}$ & - \\
\hline A0A212CL29 & large subunit ribosomal protein L5e & SD1 ${ }^{1}$ & - \\
\hline A0A212CVE3 & large subunit ribosomal protein $\mathrm{L} 4 \mathrm{e}$ & SD1 ${ }^{1}$ & - \\
\hline
\end{tabular}

\section{Extracellular Matrix Proteins}

\begin{tabular}{|c|c|c|c|}
\hline UniProt ID & Protein Name & RD2/SD1 & $P$-value \\
\hline A0A212C5S3 & laminin, alpha 4 & 0.400 & 0.0117 \\
\hline A0A212C632 & laminin, alpha $3 / 5$ & SD1 ${ }^{1}$ & - \\
\hline $\mathrm{A} 0 \mathrm{~A} 212 \mathrm{C} 6 \mathrm{~B} 1$ & collagen, type IX, alpha & SD1 ${ }^{1}$ & - \\
\hline A0A212CFD7 & collagen, type IX, alpha & SD1 ${ }^{1}$ & - \\
\hline A0A212D070 & cartilage oligomeric matrix protein & SD1 ${ }^{1}$ & - \\
\hline E7D7Z2 & Osteopontin & SD1 ${ }^{1}$ & - \\
\hline \multicolumn{4}{|c|}{ PI3K/Akt pathway } \\
\hline UniProt ID & Protein Name & RD2/SD1 & $P$-value \\
\hline A0A212C5S3 & laminin, alpha 4 & 0.400 & 0.0117 \\
\hline A0A212D1I8 & immunoglobulin heavy chain & $\mathrm{RD} 2{ }^{1}$ & - \\
\hline A0A212CMX1 & $\begin{array}{l}\text { serine/threonine-protein phosphatase } \\
2 \text { A regulatory subunit B }\end{array}$ & $\mathrm{RD} 2{ }^{1}$ & - \\
\hline A0A212C632 & laminin, alpha $3 / 5$ & SD1 ${ }^{2}$ & - \\
\hline A0A212C6B1 & collagen, type IX, alpha & SD1 ${ }^{2}$ & - \\
\hline A0A212CF73 & $\begin{array}{l}\text { guanine nucleotide-binding protein } \\
\text { subunit gamma-12 }\end{array}$ & $\mathrm{SD}^{2}$ & - \\
\hline A0A212CFD7 & collagen, type IX, alpha & SD1 ${ }^{2}$ & \\
\hline A0A212D070 & cartilage oligomeric matrix protein & SD1 ${ }^{2}$ & \\
\hline
\end{tabular}




\begin{tabular}{lll}
\hline A0A212DF15 & HSP90A & SD1 ${ }^{2}$ \\
\hline E7D7Z2 & Osteopontin & SD1 $^{2}$ \\
\hline
\end{tabular}

${ }^{1}$ Proteins are expressed exclusively in a certain antler group. 